\title{
Valuing health-related quality of life in heart failure: a systematic review of methods to derive quality-adjusted life years (QALYs) in trial-based cost-utility analyses
}

\author{
Jenny Rankin ${ }^{1}$ - Donna Rowen ${ }^{2}$ Amanda Howe ${ }^{1} \cdot$ John G. F. Cleland ${ }^{3} \cdot$ Jennifer A. Whitty ${ }^{1}$
}

Published online: 22 March 2019

(C) The Author(s) 2019

\begin{abstract}
The accurate measurement of health-related quality of life (HRQoL) and the value of improving it for patients are essential for deriving quality-adjusted life years (QALYs) to inform treatment choice and resource allocation. The objective of this review was to identify and describe the approaches used to measure and value change in HRQoL in trial-based economic evaluations of heart failure interventions which derive QALYs as an outcome. Three databases (PubMed, CINAHL, Cochrane) were systematically searched. Twenty studies reporting economic evaluations based on 18 individual trials were identified. Most studies $(n=17)$ utilised generic preference-based measures to describe HRQoL and derive QALYs, commonly the EQ-5D-3L. Of these, three studies (from the same trial) also used mapping from a condition-specific to a generic measure. The remaining three studies used patients' direct valuation of their own health or physician-reported outcomes to derive QALYs. Only 7 of the 20 studies reported significant incremental QALY gains. Most interventions were reported as being likely to be cost-effective at specified willingness to pay thresholds. The substantial variation in the approach applied to derive QALYs in the measurement of and value attributed to HRQoL in heart failure requires further investigation.
\end{abstract}

Keywords Heart failure $\cdot$ Cost utility analysis · Economic evaluation · Cost-effectiveness · QALY · Health-related quality of life

\section{Introduction}

Heart failure (HF) is common and costly to manage; it accounts for $1-3 \%$ of health care expenditure in Western Europe, North America and Latin America and causes or complicates about $5 \%$ of all US and European hospital admissions amongst adults [1]. The costs, prevalence and complexity of treating HF are increasing, along with ageing of the population. Identifying and supporting patient access to interventions that are both clinically and cost-effective are required to optimise the use of resources.

Jennifer A. Whitty

Jennifer.whitty@uea.ac.uk

1 Health Economics Group, Norwich Medical School, University of East Anglia, Norwich Research Park, Norwich NR4 7JT, UK

2 School of Health and Related Research, University of Sheffield, Sheffield, UK

3 Robertson Centre for Biostatistics \& Clinical Trials, University of Glasgow \& National Heart \& Lung Institute, Royal Brompton \& Harefield Hospitals, Imperial College, London, UK
Health-related quality of life (HRQoL) is an important outcome measure in HF that is influenced by physical, emotional or social function, role performance, pain and fatigue. There has been a drive towards patient-reported outcome measures (PROMs) and patient-reported experience measures (PREMs) in health care systems, including the British NHS. Thus, the accurate measurement and valuation of HRQoL and its response to therapy are essential for choosing treatments and allocating resources. When considering the costeffectiveness of interventions, the quality-adjusted life year (QALY) is the outcome of choice for most decision-making bodies (such as the National Institute for Health and Care Excellence, NICE in the UK [2]), as it accounts for both HRQoL and survival, and their changes, in a single metric. QALYs are typically obtained from generic preference-based measures (PBMs) such as the EQ-5D (three- or five-level version) to provide utility values and these are multiplied by the duration lived in a health state. PBMs describe HRQoL as a series of health states and then assign a utility weight to each health state on a common scale, according to the preferences of members of the public for being in different health states. An alternative method for generating utility values is the direct 
elicitation of utilities from the patients themselves, using valuation methods such as the time trade-off (TTO), standard gamble (SG) or discrete choice experiment (DCE). QALY gains can subsequently be compared between interventions for use in economic evaluation.

Accurate measurement and valuation of HRQoL relies on the availability of a PBM that is sensitive to change. Generic PBMs are commonly used in economic evaluation, and there is evidence to support the validity and reliability of the commonly used EQ-5D-3L in cardiovascular disease, particularly in moderate to severe health states [3]. However, generic measures may lack sensitivity to change as they do not capture important symptoms of HF such as breathlessness, loss of self-control and tiredness [4, 5]. Condition-specific measures of HRQoL such as the Minnesota Living With Heart Failure (MLWHF) questionnaire and Kansas City Cardiomyopathy Questionnaire (KCCQ) capture these symptoms. The KCCQ has been reported to be more sensitive than the generic EQ-5D-3L and Short-Form Survey (SF)-12 measures, particularly for detecting small rather than large changes in disease severity $[3,4,6,7]$. Consequently, researchers have called for the inclusion of condition-specific measure alongside generic measures when capturing effectiveness for these conditions [8].

Whilst condition-specific measures are sensitive in capturing HRQoL, none of the available condition-specific measures in HF are preference-based [9]. Therefore, they cannot be used directly to generate QALYs. Other approaches such as mapping have been used to generate utility weights where no PBM was used, meaning a HFspecific measure could be used to measure HRQoL and this could be mapped to a measure such as the EQ-5D to generate utility weights. However, mapping is only appropriate if both measures are appropriate for the patient population, and relies on overlap between the two measures. Any symptoms captured in the HF-specific measure are unlikely to feature in the mapping model, meaning the sensitivity of the HF-specific measure to change is not necessarily maintained when mapped to EQ-5D. Therefore, the lack of availability of utility indices for condition-specific measures is likely to limit their use in the economic evaluation of HF interventions.

Accordingly, we conducted a systematic review to identify and describe the approaches used to measure and value change in HRQoL in trial-based economic evaluations of HF interventions which derive QALYs as an outcome measure. We sought to investigate the extent to which utility weights are generated using different approaches: generic PBM, mapping to a PBM or directly ascertained using a valuation method. A secondary objective was to identify whether published papers reported whether interventions for HF were cost-effective.

\section{Methods}

\section{Protocol and registration}

The review protocol is registered at the International Prospective Register of Systematic Reviews (PROSPERO) and can be accessed at: https://www.crd.york.ac.uk/prospero/ display record.php? RecordID $=78519$ registration number CRD42017078519.

\section{Eligibility criteria}

The inclusion and exclusion criteria are presented in Table 1. Randomised controlled trials that were published in full, in English, and compared costs and benefits expressed as QALYs as an outcome measure were included if they evaluated an intervention designed to investigate the treatment or management of HF in adults ( $\geq 18$ years old). There were no upper age limit, sex or publication date restrictions. Studies that included participants without HF, systematic reviews, modelled studies, meta-analyses and those published as abstract only were excluded.

\section{Search strategy}

PubMed Central, Cumulative Index to Nursing and Allied Health Literature (CINAHL) and the Cochrane Library (NHS Economic Evaluation Database) were searched between 26 June and 3 July 2017, with no date restrictions.

The following search terms were used:

PubMed Central: "heart failure"[Abstract] AND (cost utility analysis [Abstract] OR CUA [Abstract] OR economic evaluation [Abstract] OR cost effectiveness [Abstract])

Cochrane Library: "heart failure" AND (cost utility analysis OR CUA OR economic evaluation OR cost effectiveness)

CINAHL: "heart failure" AND (cost utility analysis OR CUA OR economic evaluation OR cost effectiveness)

\section{Study selection}

The results from the databases were combined, and duplicates were removed. Papers were screened on their title and abstract. Potentially relevant papers were retrieved in full and screened against the inclusion/exclusion criteria. Bibliographies of relevant papers were hand-searched for any sources potentially missed within the database searches. 
Table 1 Inclusion and exclusion criteria

\begin{tabular}{|c|c|}
\hline Inclusion criteria (if all of the following met) & Exclusion criteria (if any of the following met) \\
\hline 1. Original research & 8. Papers other than in the English Language \\
\hline $\begin{array}{l}\text { 2. Adults (aged } 18 \text { and over) } \\
\text { of any sex or ethnic group }\end{array}$ & $\begin{array}{l}\text { 9. Design/protocol papers, systematic reviews, } \\
\text { meta-analyses and commentaries/editorials }\end{array}$ \\
\hline $\begin{array}{l}\text { 3. Interventions designed to treat } \\
\text { or manage heart failure }\end{array}$ & $\begin{array}{l}\text { 10. Effectiveness estimates not based } \\
\text { on actual trial data (e.g. hypothetical intervention } \\
\text { or summarised effect) }\end{array}$ \\
\hline $\begin{array}{l}\text { 4. Trial-based analyses based on data } \\
\text { from randomised control trials }\end{array}$ & $\begin{array}{l}\text { 11. Trials investigating conditions other } \\
\text { than heart failure }\end{array}$ \\
\hline 5. Comparison of costs and benefits expressed as QALYs & 12. Studies published as abstract only \\
\hline 6. Published papers in English & 13. Prevention and diagnostic interventions \\
\hline 7. Participants with heart failure & 14. Model-based studies \\
\hline
\end{tabular}

\section{Data extraction}

The characteristics and results of relevant studies were extracted based on an amended version of a standardised data collection form recommended by the Cochrane Training (Cochrane Library: http://training.cochrane.org/resource/ data-collection-forms-intervention-reviews). The form was amended to collect characteristics relevant to describe the economic evaluation and HRQoL measure and valuation.

The following were extracted:

- Measurement and valuation of HRQoL including measure and approach used to generate utility weights

- The elicitation method, tariff and population used to derive the utility weights used to value HRQoL

- The mean difference in QALYs between the intervention and control groups from baseline to follow-up with significance levels and confidence intervals (if available)

- Incremental cost per QALY of intervention(s) and indication of the level of uncertainty (such as confidence interval) around that estimate

- Probability of cost-effectiveness at a specified threshold

Where a study cited other papers as the source of the utility weights to derive QALYs, the original source of utility weights was retrieved to enable a description of the approach to deriving QALYs.

\section{Quality assessment}

The Consolidated Health Economic Evaluation Reporting Standards (CHEERS) statement was used to assess the reporting quality of studies [10]. Each of the 24 items in the CHEERS checklist was assigned a weight ranging from zero to two (representing studies that did not report, reported poorly or reported well) which were used to calculate an average reporting quality score.

\section{Results}

\section{Study selection}

A total of 492 citations were identified by the database searches, with 11 additional citations identified by hand searching (Fig. 1). Twenty studies were included in the final review (Table 2).

\section{Study characteristics}

The 20 papers included within the review were trial-based evaluations based on data sourced from 18 individual

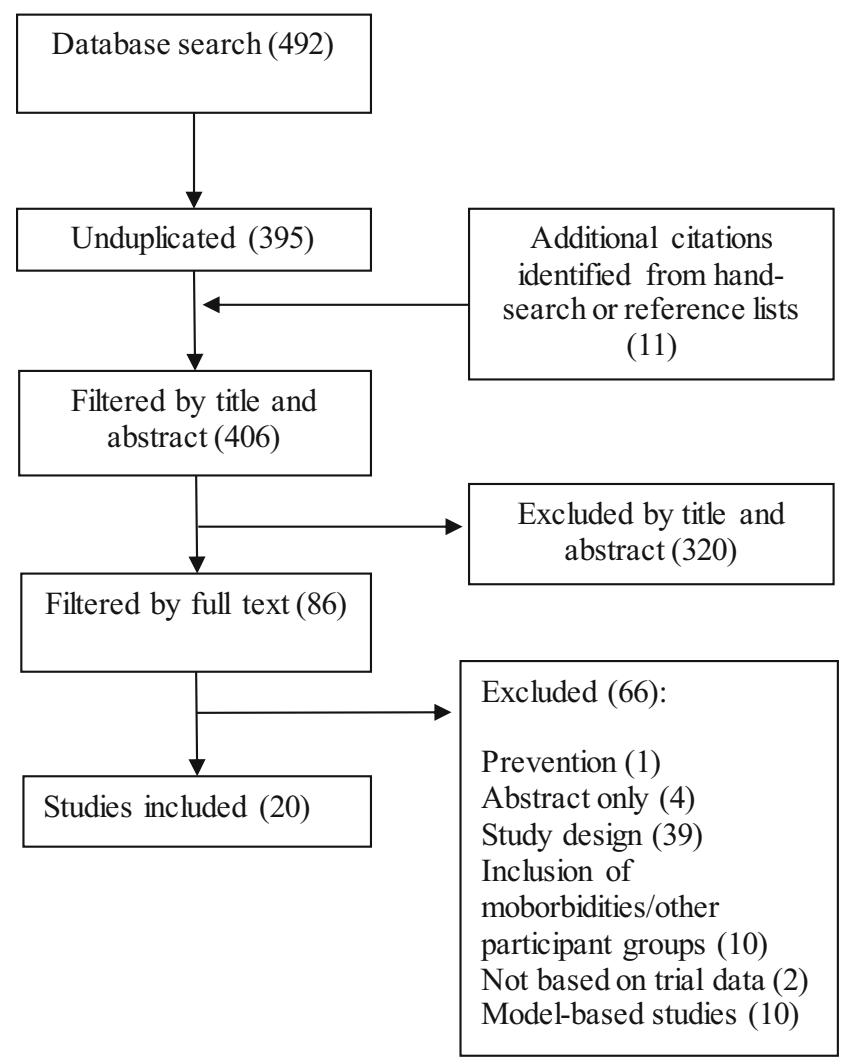

Fig. 1 Study selection 


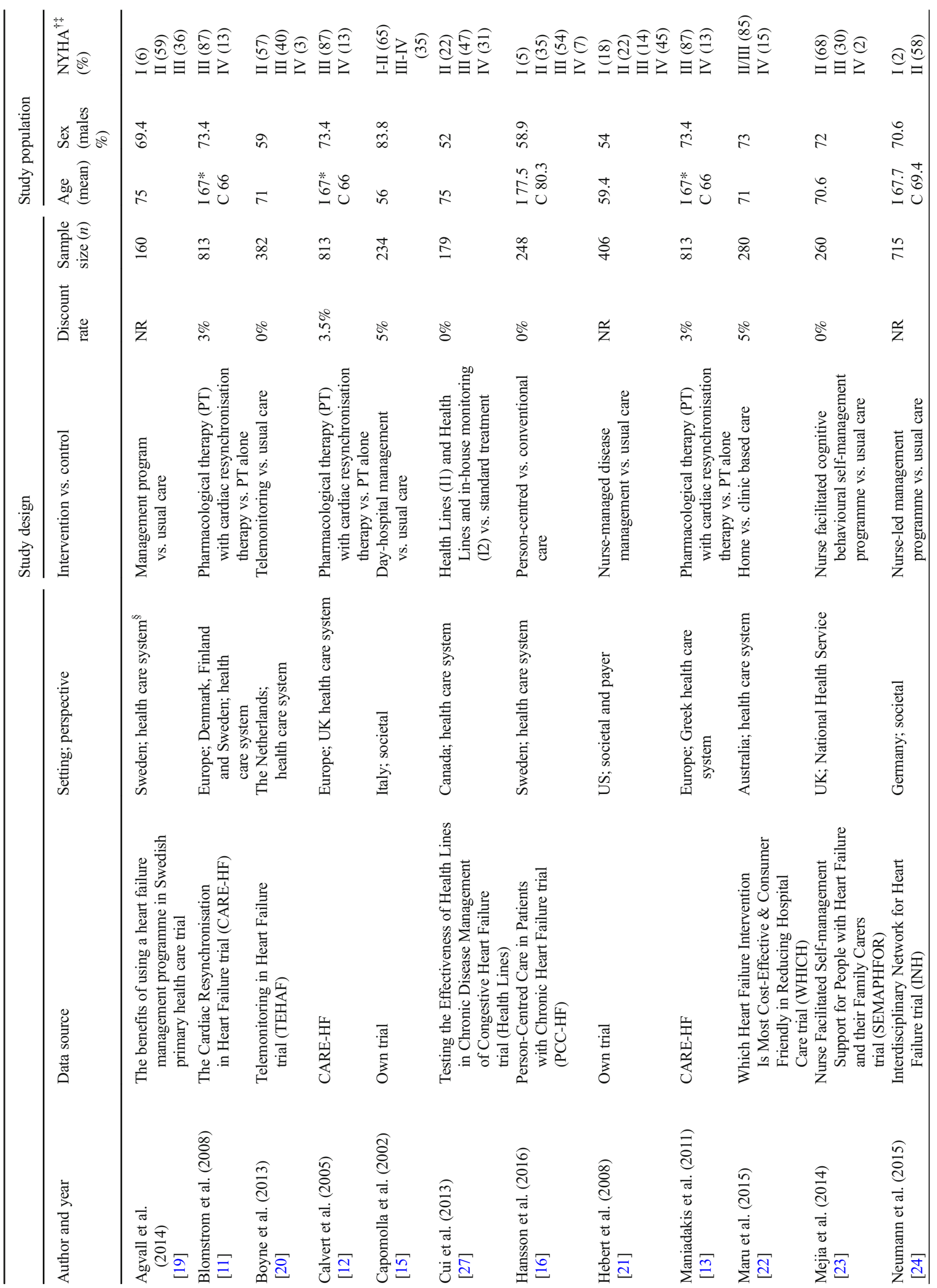




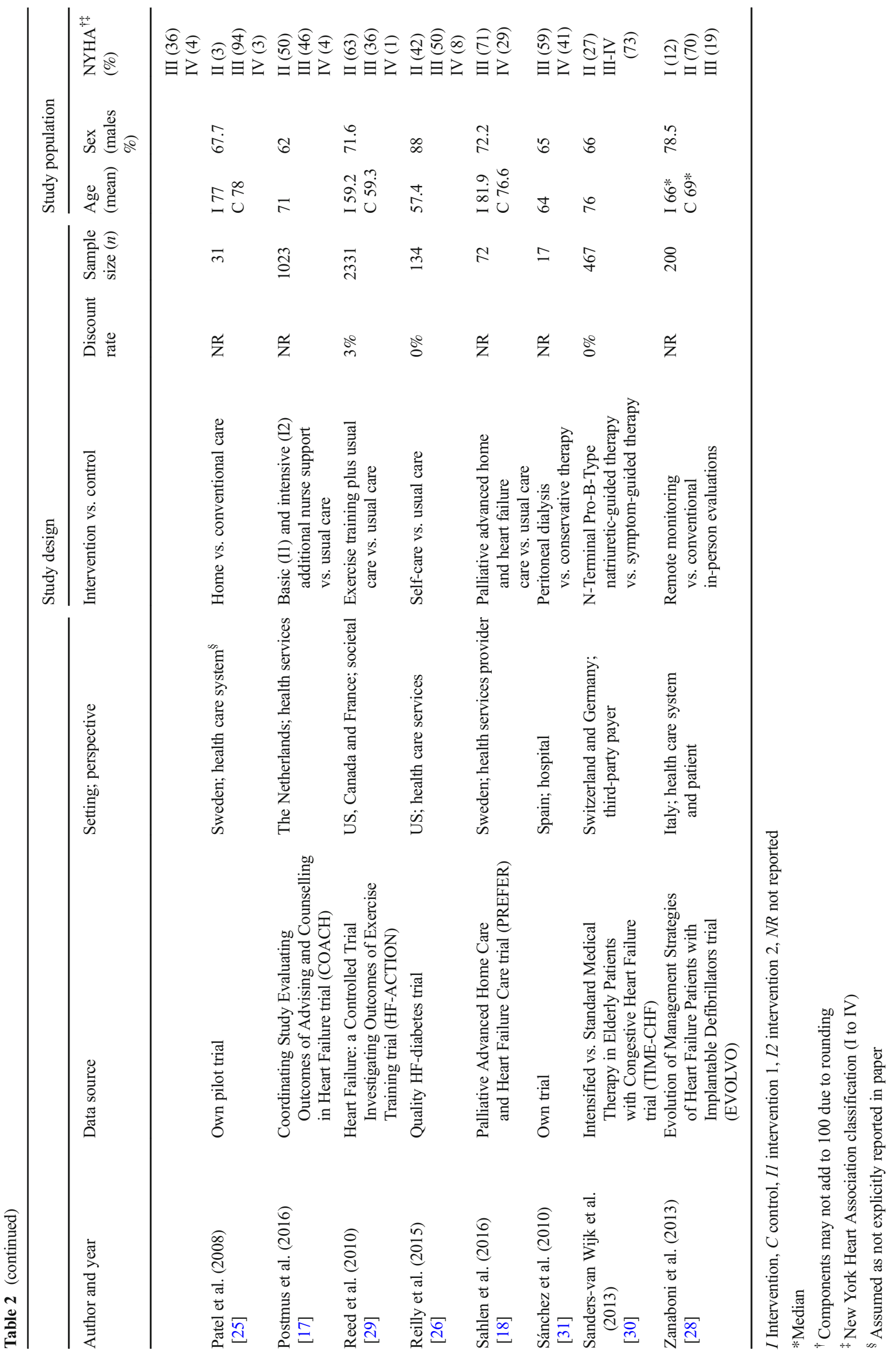


randomised controlled trials (RCT), with three papers [11-13] based on the Cardiac Resynchronisation in Heart Failure (CARE-HF) trial [14]. The date of publishing ranged from 2002 [15] to 2016 [16-18].

The majority of papers $(n=16)$ focused on management interventions as opposed to treatment $(n=4)$ for HF. Management interventions included the following: nurse led [17, 19-26], telemonitoring [27, 28], outpatient clinic based [15], personcentred care [16, 18], exercise training [29] and NT-proBNPguided therapy [30]. Treatment interventions included cardiac resynchronisation therapy [11-13] and peritoneal dialysis [31].

Most papers adopted the perspective of their respective countries' health care system or third-party payers, and one study [28] included the costs and effects from a patient perspective. The remaining studies adopted a societal [15, 24, 29], a combination of both a societal and payer [21] or a single hospital perspective [31]. The study perspective was not explicitly reported in two papers [19,25]; a health care system perspective was assumed in both because of the costings involved within the trials.

Sample sizes within the trials ranged from 17 [31] to 2331 [29] with a total of 7952 participants across all studies (accounting for the individuals in the CARE-HF trial only once). The overall population studied within the trials was predominantly men $(68.2 \%)$ with mean/median ages ranging from $56 \pm 10$ [15] to $81.9 \pm 7.2$ years [18]. Fifteen papers reported average participant ages of $>60$ years. Three papers excluded participants based on age: those aged under 21 (with an age limit of 82) [26], under 40 [27] and under 60 years [30].

New York Heart Association (NYHA) functional class, a commonly used physician-assessed tool measuring patient's functional ability in HF, was reported in all papers reviewed. Seventeen trials reported individual participant category numbers for each NYHA class (NYHA classes were grouped in three studies $[15,22,30]$ and therefore were not included in the following summary). Most participants were assigned to NYHA class II (47.5\%) and III (43.7\%) rather than class IV (the most severe; $6.9 \%$ ) or class I (asymptomatic; $1.9 \%$ ). Overall, eight reports had exclusion criteria in relation to participant NYHA class, with five studies excluding NYHA class I $[22,25,26,29,30]$ and CARE-HF (three reports) excluded NYHA class I and II [11-13].

The discount rate used for assessing costs and effects in the trial-based economic evaluations ranged from to $0 \%[16,20$, $23,26,27,30]$ in the studies who had a follow-up of less than 12 months to $3 \%[11,13,29], 3.5 \%$ [12] and $5 \%[15,22]$. No discount rate was reported in eight studies [17-19, 21, 24, 25, $28,31]$.

\section{Measurement and valuation of HRQoL to derive QALYs}

A summary of the approach used to derive QALYs in the base-case analyses for the 20 studies is provided in Table 3 .

\section{Description of health states used to derive QALYs}

Most ( $n=17$ ) of the studies used a validated, generic PBM of HRQoL completed by participants to describe the health status of participants. The EQ-5D was the most commonly used $(n=13)$, with most studies using the $3 \mathrm{~L}$ version. Two studies did not indicate the version of EQ-5D used (3L or 5L) $[24,28]$ but seem likely to have used the $3 \mathrm{~L}$ version based on publication date (2013 and 2015). Two studies used SF-36, and two studies used SF-12 to describe participants' health states. Three of the 17 studies which used a generic PBM also used the MLWHF condition-specific questionnaire to collect longer term (median 29.4 month) follow-up data to describe HRQoL for the purpose of deriving QALYs in a cost-utility analysis that extended outcomes beyond the end of the initial trial. These three cost-utility analyses (CUAs) were all undertaken alongside the CARE-HF trial [11-13]. Of the remaining three studies, two studies used patients own perceived health states to derive QALYs $[15,25]$, one of which also described health status using the EQ-5D visual analogue scale (VAS) [25]. The final study [19] used the physician-reported NYHA assessment to describe health status [32].

\section{Valuation of HRQoL}

Of the 17 studies using a generic PBM to describe health status of the patients in their trials, most $(n=13)$ reported that they applied an existing utility tariff for valuation. For the 13 studies using EQ-5D, eight studies applied the UK tariff in which the EQ-5D-3L health states were valued by a sample of the public using TTO methods, one study applied Australian TTO weights, and one study applied the European EQ-net weights, in which health states were valued using VAS methods. The remaining three studies using EQ-5D to describe health status did not clearly report the method for deriving utilities [18, 24, 31]; although, in two studies, the German and Spanish tariffs based on TTO methods would appear to have been used to assign utility weights to the EQ$5 \mathrm{D}$ health states $[24,31]$. Both studies using the SF-36 and one of the studies using the SF-12 to describe participants' health status applied the SF-6D algorithm to assign utilities. The SF-6D algorithm was developed based on the preferences of a UK public sample for being in different health states, using the SG valuation method [34]. The final study using the SF-12 to measure participant health status [21] mapped SF-12 results onto the Health Utilities Index Mark 3 (HUI-3) and the EQ-5D-3L in two separate base case analyses using a conversion formula based on the results from a low-income minority population [35].

The three studies which used the MLWHF questionnaire, a condition-specific HRQoL instrument for which there is no utility tariff available, to both describe and value HRQoL alongside the EQ-5D-3L, were CUAs undertaken alongside 


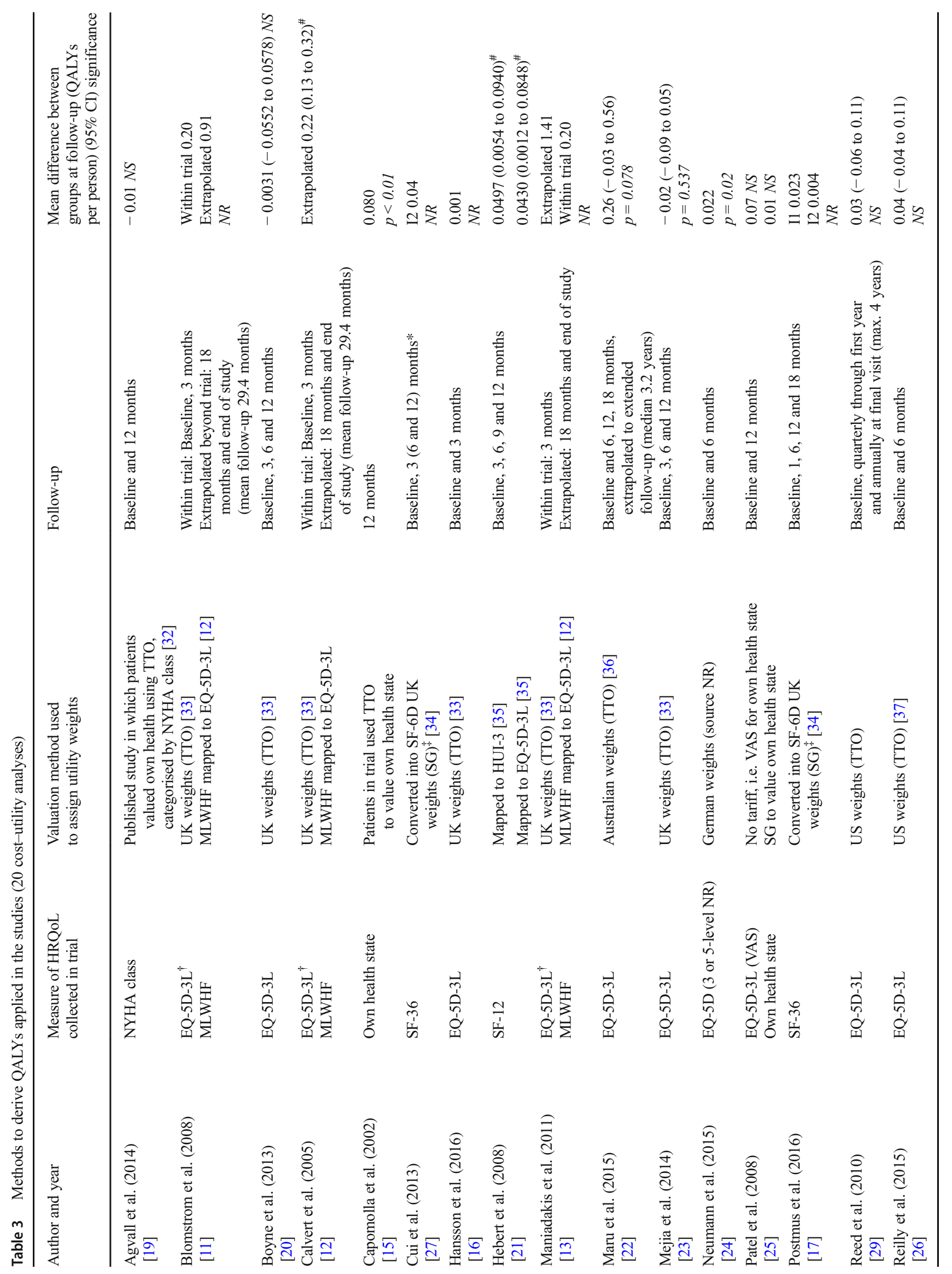




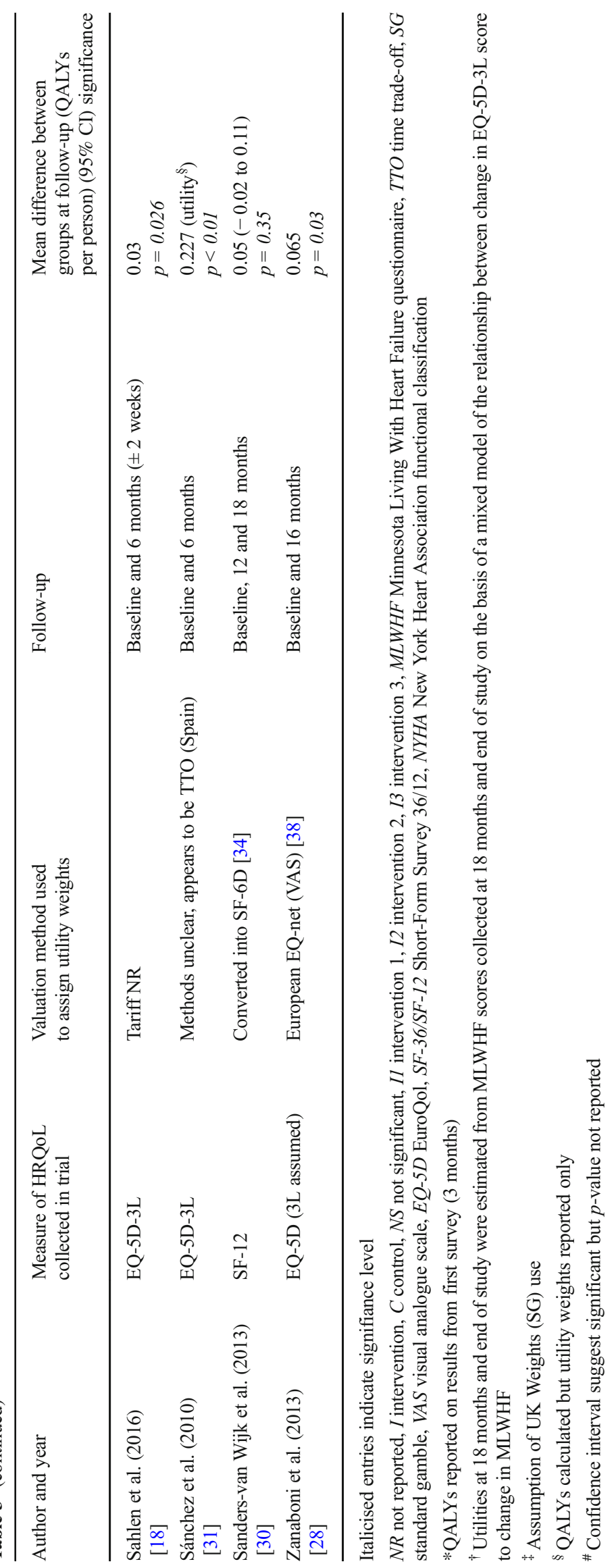


the CARE-HF trial [11-13]. They used the MLHF data collected at a median of 29.4 months follow-up to model utility outcomes beyond the initial period of the trial. The authors assigned utility weights to model utility outcomes beyond the initial period of the trial based on a mixed model mapping the relationship between change in EQ-5D-3L and change in MLWHF which were both completed at baseline and 90 days follow-up in the trial [12].

Of the remaining three studies, two studies used patients' direct valuation of their own health states to assign utility weights (one using the TTO approach [15] and one using the EQ-5D VAS and SG approaches [25]). The final study in which participants health status was described using NYHA [19] assigned utility weights to NYHA classes using a published study in which elderly patients with heart failure valued their own health status using TTO methods [32].

\section{Change in QALY reported by the studies}

We attempted to examine whether the evaluations undertaken alongside trials identified significant changes in QALYs (Table 3). Only seven of the 20 analyses undertaken alongside trials reported significant incremental QALY gains, ranging between 0.022 and 0.22 QALYs per person over follow-up periods of up to a mean of 29.4 months. Interestingly, none of these generated utility weights using the combination of EQ5D-3L with the UK TTO tariff, despite this being the most common approach to generate utility weights across the studies. Instead, the seven studies [12, 15, 18, 21, 24, 28, 31] reporting significant QALY differences used the EQ-5D-3L with US, Spanish or European utility weights, the EQ-5D (3L/ $5 \mathrm{~L}$ not stated) with German weights, the MLWHF mapped to EQ-5D-3L, SF-12 mapped to EQ-5D/HUI-3 or direct TTO valuation by patients to derive QALYs. Eight analyses [19, 20, 22, 23, 25, 26, 29, 30] did not report significant QALY differences between interventions. Two of these used the EQ5D-3L to describe health states combined with the UK tariffs, others used US ( $n=2)$ or Australian $(n=1)$ tariffs to value the gain, one used the SF-12 converted to SF-6D utility weights, one used the EQ-5D-3L VAS scale to derive patient's direct valuations for health states and another used NYHA class with utility weights derived from TTO from previous literature. Cost-utility analyses of five trials $[11,13,16,17,27]$ did not report the significance of any change in QALY.

\section{Cost-effectiveness of interventions}

Table 4 summarises the overall findings of the 20 studies with respect to the cost-effectiveness of interventions evaluated for the management or treatment of heart failure. Overall, most interventions were reported as being cost-effective using the thresholds the studies applied to their own evaluations (which were dependent upon country of study and relevant international agency). The incremental cost-effectiveness ratios (ICERs) ranged from a cost-saving of $-61,081 €[31]$ to $98,000 €[16]$ per QALY gained. Probabilities of being costeffective ranged from 0.08 at a $20,000 €$ threshold [39] to around 1.0 at a $25,000 €$ threshold [13]. Eight interventions were reported as being dominant $[17,18,22,26,28-31]$ compared to the control group, and one was reported as being dominated [23]. Two studies did not publish cost per QALY, and seven studies did not publish a cost-effectiveness probability estimate $[15,16,18,19,25,28,31]$.

\section{Quality of reporting (CHEERS checklist)}

Table 4 shows scores for the CHEERS checklist, reported as a percentage (\%) out of a maximum score of 48 . The quality of reporting of the studies ranged from $62.5 \%$ [25] (probably due to the small scale of this pilot study) to $97.9 \%$ [23].

\section{Discussion}

Although HF is associated with a large health care burden, this systematic review identified rather few valid health economic analyses of relevant RCTs suggesting that many economic evaluations of HF interventions do not consider HRQoL as an outcome measure. For example, a search of the NHSEED (National Health Service Economic Evaluation) database which holds comprehensive records of published health economic evaluations identified 178 records with the term "heart failure" or "cardiac failure" in the title (database available at https://www.crd.york.ac.uk/CRDWeb/ search performed 4 January 2018). Moreover, Goehler et al. (2011) reviewed 34 decision-analytic modelled studies in HF, in which only 19 reported QALYs as an outcome measure. Nevertheless, most (though not all) of the cost-utility analyses identified in this review followed what is currently regarded as "best practice" for the derivation of QALYs and their consideration in economic evaluation [40, 41]. That is, they adopted generic measures, notably the EQ-5D-3L and SF-36 and its derivative (SF-12) on which participating patients describe their own health states, and then the preferences of a general population sample were used to value improvements in HRQoL by assigning utility weights from existing tariffs. The high frequency with which the EQ-5D-3L was used is consistent with the dominance of EQ-5D-3L in other clinical areas and the prescriptive guidance from NICE requiring EQ-5D-3L to generate utility weights in health technology assessments [2].

The methods used to derive QALYs in the identified studies were variable, however. In particular, they raise questions in the context of heart failure, around several methodological issues that are debated in the health state valuation literature. To undertake cost-utility analyses utilising the QALY as an 


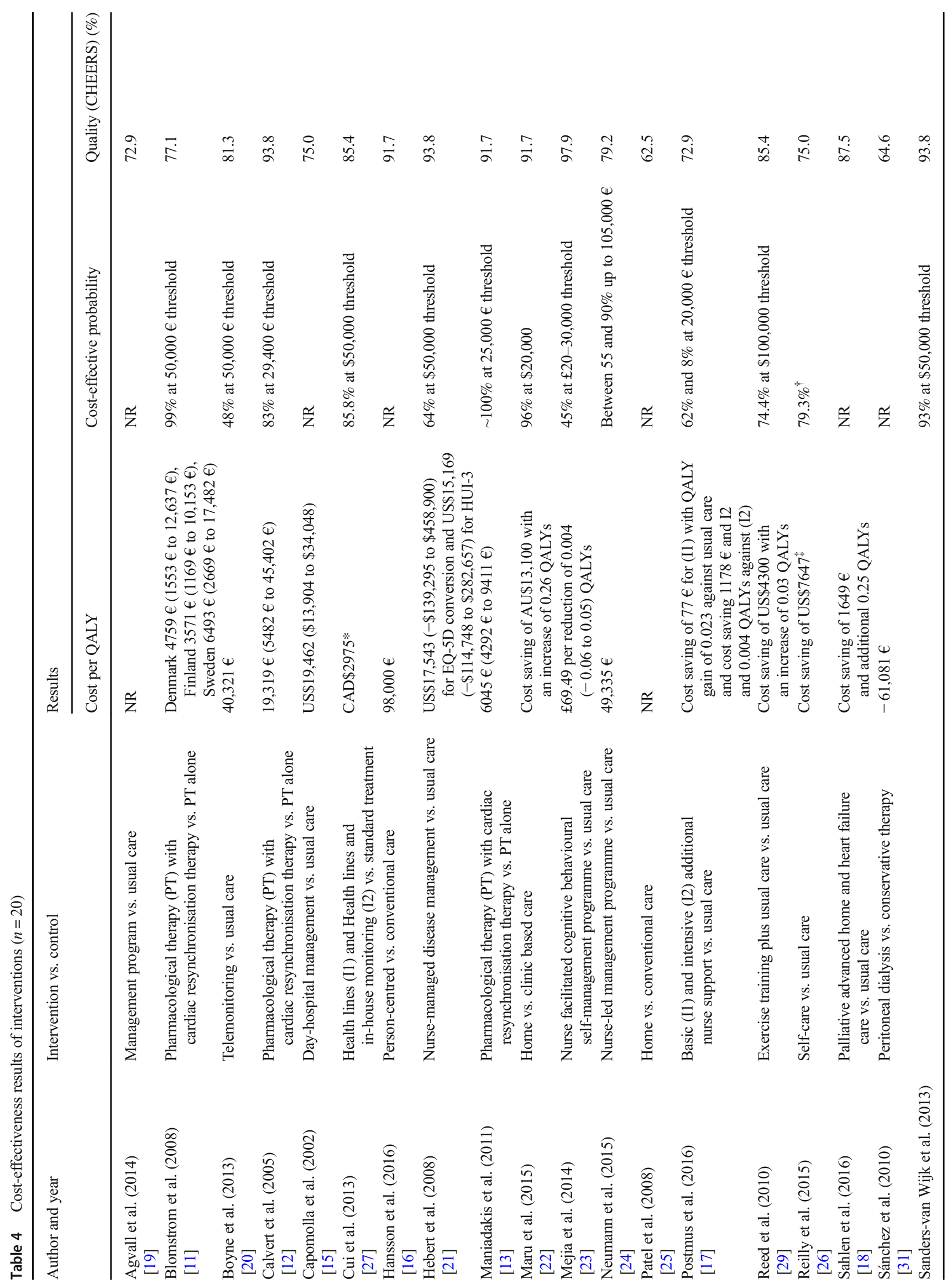


outcome, it is necessary to both accurately measure change in HRQoL and to appropriately assign the utility value associated with that change using public preferences. Only generic PBMs which are designed to be used across a range of different conditions (such as the EQ-5D) are available as validated instruments to both measure and value HRQoL in HF. These have the advantage that if they are used consistently, they provide a common approach to measurement and valuation of HRQoL across all conditions, thus allowing a direct comparison of the benefit of allocating resources to heart failure alongside the benefit of allocating resources to address other health conditions. Condition-specific measures of HRQoL are more sensitive to change in HRQoL in HF, but existing instruments are not preference-based and so do not have a utility valuation tariff to derive QALYs $[4,6,7,42]$. This might explain why only three cost-utility analyses (based on a single trial) attempted to use condition-specific HRQoL data to derive QALYs. The need for sensitive condition-specific measures to be used alongside generic measures to ensure the accurate capture and valuation of change in HRQoL has been raised previously across a range of clinical areas [5], including HF [43]. Indeed some international reimbursement agencies, such as NICE, allow the inclusion of analyses using conditionspecific measures in sensitivity analyses to support the appraisal of health care interventions [2]. However, none of the studies identified in this review tested the impact on the costeffectiveness estimates of using weights based on a conditionspecific preference-based measure as opposed to a generic preference-based measure in the evaluation. Therefore, we are unable to provide further evidence to inform the debate on the relative value of generic vs. condition-specific measures in the setting of heart failure.

This review found mixed results in QALY outcomes; with similar numbers of studies finding significant, non-significant or unreported significance in differences between interventions in QALYs. This may be because the intervention was not effective, has a problem with trial size or design or the failure of the HRQoL tool to accurately capture change. Some measures used may not be responsive to changes in HRQoL. This would not be surprising as some trials failed to show improvements in HRQoL despite reductions in mortality and hospital readmission [44]. Alternatively, it may be that any change in HRQoL is captured, but the improvements in HRQoL are not considered meaningful according to the preferences of members of the public when they are valued and converted to QALYs. Within the review, a pilot study [25] used both the EQ-5D VAS and the SG to derive utility weights for the intervention and control groups in the same sample, giving a different mean difference in QALYs between groups across the two methods, suggesting they do not measure or value HRQoL in the same way as would be expected. This threatens the convergent validity of the VAS and SG methods to value change in HRQoL within the HF population. This 
lack of concordance between VAS, TTO and SG has been widely reported outside of HF [45]. Although most studies in the review used valuation tariffs that were derived using TTO methods, some used SG or VAS approaches to derive utility weights. The variation in methods for deriving QALYs identified in this review is therefore a concern and potentially threatens the consistency of the evidence on any decisions based on the findings of these evaluations. Nevertheless, the interventions reported generally appeared to be cost-effective when compared to specified decision-making thresholds for willingness to pay for a QALY gain. Therefore, it is possible that the variable statistical significance of the QALY gains identified in this review and the possible suboptimal sensitivity of some approaches to measuring and valuing HRQoL may not alter decision-making. However, reporting bias is also possible; interventions that are cost-effective are more likely to be published.

One possible solution to lack of sensitivity to change in generic PBM has been to measure change in HRQoL using a condition-specific measure and then to map these back to a generic PBM in order to generate utility weights $[9,46]$. However, this may not maintain the sensitivity of the condition-specific measure if the generic PBM is not sensitive to these symptoms. This mapping approach was only observed within this review for the CARE-HF trial [11-13]. It has also been undertaken in several model-based studies in HF $[39,47,48]$ but has received criticism regarding the potential error component in the algorithm used to map utility weights to the MLWHF [47]. Mapping is not a panacea and has been considered theoretically inferior $[40,46]$. For example, the conversion of generic SF-12 scores into two different measures to derive QALYs in one study in this review [21] resulted in two different mean difference values between groups at follow-up, likely because the EQ-5D-3L does not contain a dimension for energy or vitality, leading to small and nonsignificant coefficients when mapped and potentially erroneous mapping values [49]. One study [19] within the review used a physician-reported measure, the NYHA to calculate QALYs by using a utility weighting from previous literature [32], which centres on domains of physical health and functional status as opposed to HRQoL. There is inherent uncertainty associated with both designating an NYHA class and the algorithm used to estimate utility weights, and it may not fully capture individuals' HRQoL [50]. The three studies mapping MLWHF to EQ-5D-3L for the CARE-HF trial [11-13] identified in the review and several modelling studies $[39,51]$ all used the same algorithm reported by Calvert et al. [12]. It is unclear whether the method of estimation of weights on the basis of a mixed model relationship between the change in the EQ-5D-3L and MLWHF change is robust or not in capturing and modelling HRQoL changes. Standard guidelines exist for best practice methods in mapping studies [52-54], but the mapping algorithm utilised cannot be assessed against these as it has not been fully published or peer reviewed, only appearing in summary form in a cost utility analysis publication [12].

All of the studies identified in this review used the trial participants to describe their own health state for valuation, mostly via completion of a HRQoL instrument or a visual analogue scale (although, one study used physician assessment of NYHA class rather than the patient's own perception of their health state as the basis for valuation). However, not all studies assigned the preferences of a general public sample to derive the valuation. Three studies used the preferences of patients with heart failure as the basis for the utility weights used to derive QALYs [15, 19, 25]. Arguments have been advanced in the literature both for and against the use of patient as opposed to public preferences for the valuation of health states [55-57]. However, consensus is generally aligned with the use of public preferences where the purpose of an evaluation is to inform resource allocation cross the health system [40], and NICE requires the use of public rather than patient preferences in their reference case [2].

\section{Limitations}

Whilst we were inclusive in our approach to selecting studies, the diversity of the literature and necessity for narrow search terms may mean that some potentially relevant papers were missed. We reduced this risk by hand-searching identified papers. Publication bias is likely, but this is beyond the control of a systematic review. A language bias is also possible, as only published in English were retrieved. Our review only included trials which reported QALYs; thus, it does not present a complete picture of how HRQoL is measured or reported in heart failure. Trials that may have measured HRQoL but not derived QALYs, measured other aspects of the "patient journey" (e.g. the COMET study [58]) or studies reporting QALYs but principally using a modelling approach were excluded. This was a purposeful decision, since our focus was on how HRQoL was valued and QALYs were derived in primary research in heart failure.

Most participants included in this review were older men in NYHA class II or III, which is consistent with the majority of those enrolled in RCTs of HF. Limitations at the study and outcome level include the amount of missing HRQoL data, with some studies reporting $22-23 \%$ [16], 37\% [30] and $12 \%$ [28] of participants with incomplete data. Approaches for dealing with missing data included the use of last-observation-carriedforward [11, 13, 22, 29] and imputation methods [20, 21, $23,28]$, both of which have drawbacks in measuring HRQoL in the long term. In clinical trials where mortality is high and a utility weight of zero is assigned from the time of death, this may make a large contribution to the QALY value. 


\section{Conclusions}

Comparatively, few economic evaluations undertaken alongside clinical trials in patients with HF report QALY as an outcome measure. This is unfortunate given the importance of HRQoL (in addition to survival) as a treatment goal, both from a patient and health care professional's perspective and for the determination of cost-effectiveness. This review suggests that the published evidence on cost-effectiveness that might underpin decisions regarding resource allocation for HF interventions is based on a variety of methodological approaches and usually relies on the sensitivity of generic measures. A review nearly 20 years ago suggested the optimal method of assessing HRQoL was a combination of both generic and condition-specific measures [8]. Findings suggest there has been no substantial progress in the most effective way to measure and value HRQoL for the purpose of deriving QALY outcomes in people with HF.

\section{Key points}

- The accurate valuation of HRQoL is important to inform resource allocation decisions.

- We found few economic evaluations undertaken alongside clinical trials in heart failure have reported QALYs as an outcome.

- Trial-based economic evaluations have generally used generic measures of HRQoL to derive QALYs, but there is substantial variation in approach.

- Less than half the studies identified reported significant QALY gains between intervention groups

\section{Compliance with ethical standards}

Conflict of interest JGFC has received research grants and speakers honoraria from Amgen, Bayer, Medtronic, Novartis and Servier. JR, $\mathrm{DR}, \mathrm{AH}$ and JAW are not aware of any potential conflicts of interest related to the review.

Open Access This article is distributed under the terms of the Creative Commons Attribution 4.0 International License (http:// creativecommons.org/licenses/by/4.0/), which permits unrestricted use, distribution, and reproduction in any medium, provided you give appropriate credit to the original author(s) and the source, provide a link to the Creative Commons license, and indicate if changes were made.

\section{References}

1. Ponikowski P, Anker SD, AlHabib KF, Cowie MR, Force TL, Hu S, Jaarsma T, Krum H, Rastogi V, Rohde LE, Samal UC, Shimokawa H, Budi Siswanto B, Sliwa K, Filippatos G (2014) Heart failure: preventing disease and death worldwide. ESC Heart Fail 1(1):4-25. https://doi.org/10.1002/ehf2.12005
2. NICE (2013) Guide to the methods of technology appraisal. National Institute for Health and Clinical Excellence (NICE), London

3. Dyer MT, Goldsmith KA, Sharples LS, Buxton MJ (2010) A review of health utilities using the EQ-5D in studies of cardiovascular disease. Health Qual Life Outcomes 8:13. https://doi.org/10.1186/ 1477-7525-8-13

4. Eurich DT, Johnson JA, Reid KJ, Spertus JA (2006) Assessing responsiveness of generic and specific health related quality of life measures in heart failure. Health Qual Life Outcomes 4:89. https:// doi.org/10.1186/1477-7525-4-89

5. Payakachat N, Ali MM, Tilford JM (2015) Can the EQ-5D detect meaningful change? A systematic review. Pharmacoeconomics 33: 1137-1154. https://doi.org/10.1007/s40273-015-0295-6

6. Green CP, Porter CB, Bresnahan DR, Spertus JA (2000) Development and evaluation of the Kansas City Cardiomyopathy Questionnaire: a new health status measure for heart failure. J Am Coll Cardiol 35:1245-1255

7. Spertus J, Peterson E, Conard MW, Heidenreich PA, Krumholz HM, Jones P, McCullough P, Pina I, Tooley J, Weintraub WS, Rumsfeld JS, Cardiovascular Outcomes Research Consortium (2005) Monitoring clinical changes in patients with heart failure: a comparison of methods. Am Heart J 150:707-715. https://doi.org/ 10.1016/j.ahj.2004.12.010

8. Berry C, McMurray J (1999) A review of quality-of-life evaluations in patients with congestive heart failure. Pharmacoeconomics 16: 247-271

9. Rowen D, Brazier J, Ara R, Azzabi Zouraq I (2017) The role of condition-specific preference-based measures in health technology assessment. Pharmacoeconomics 35:33-41. https://doi.org/10. 1007/s40273-017-0546-9

10. Husereau D, Drummond M, Petrou S, Carswell C, Moher D, Greenberg D, Augustovski F, Briggs AH, Mauskopf J, Loder E, ISPOR Health Economic Evaluation Publication GuidelinesCHEERS Good Reporting Practices Task Force (2013) Consolidated Health Economic Evaluation Reporting Standards (CHEERS) - explanation and elaboration: a report of the ISPOR Health Economic Evaluation Publication Guidelines Good Reporting Practices Task Force. Value Health 16:231-250. https:// doi.org/10.1016/j.jval.2013.02.002

11. Blomström P, Ekman M, Lundqvist CB, Calvert MJ, Freemantle N, Lönnerholm S, Wikström G, Jönsson B (2008) Cost effectiveness of cardiac resynchronization therapy in the Nordic region: an analysis based on the CARE-HF trial. Eur J Heart Fail 10:869-877

12. Calvert M, Freemantle N, Yao G, Cleland JG, Billingham L, Daubert JC, Bryan S, CARE-HF investigators (2005) Costeffectiveness of cardiac resynchronization therapy: results from the CARE-HF trial. Eur Heart J 26:2681-2688

13. Maniadakis N, Ekman M, Calvert MJ, Freemantle N, Karamalis M, Vardas P (2011) Cost effectiveness of cardiac resynchronization therapy in Greece: an analysis based on the CArdiac REsychronization in Heart Failure trial. Europace 13:1597-1603. https://doi.org/10.1093/europace/eur188

14. Cleland J, Daubert JC, Erdmann E, Freemantle N, Gras D, Kappenberger L, Tavazzi L, Cardiac Resynchronization-Heart Failure (CARE-HF) Study Investigators (2005) The effect of cardiac resynchronization on morbidity and mortality in heart failure. N Engl J Med 352:1539-1549

15. Capomolla S, Febo O, Ceresa M, Caporotondi A, Guazzotti G, la Rovere MT, Ferrari M, Lenta F, Baldin S, Vaccarini C, Gnemmi M, Pinna GD, Maestri R, Abelli P, Verdirosi S, Cobelli F (2002) Cost/ utility ratio in chronic heart failure: comparison between heart failure management program delivered by day-hospital and usual care. J Am Coll Cardiol 40:1259-1266

16. Hansson E, Ekman I, Swedberg K, Wolf A, Dudas K, Ehlers L, Olsson LE (2016) Person-centred care for patients with chronic 
heart failure - a cost-utility analysis. Eur J Cardiovasc Nurs 15: 276-284

17. Postmus D, Abdul Pari AA, Jaarsma T, Luttik ML, van Veldhuisen DJ, Hillege HL, Buskens E (2011) A trial-based economic evaluation of 2 nurse-led disease management programs in heart failure. Am Heart J 162:1096-1104

18. Sahlen KG, Boman K, Brannstrom M (2016) A cost-effectiveness study of person-centered integrated heart failure and palliative home care: based on a randomized controlled trial. Palliat Med 30:296-302. https://doi.org/10.1177/0269216315618544

19. Agvall B, Paulsson T, Foldevi M, Dahlstrom U, Alehagen U (2014) Resource use and cost implications of implementing a heart failure program for patients with systolic heart failure in Swedish primary health care. Int J Cardiol 176:731-738. https://doi.org/10.1016/j. ijcard.2014.07.105

20. Boyne JJ, di van Asselt A, Gorgels APM, Steuten LMG, de Weerd G, Kragten J, Vrijhoef HJM (2013) Cost-effectiveness analysis of telemonitoring versus usual care in patients with heart failure: the TEHAF-study. J Telemed Telecare 19:242-248. https://doi.org/10. 1177/1357633X13495478

21. Hebert PL, Sisk JE, Wang JJ, Tuzzio L, Casabianca JM, Chassin MR, Horowitz C, McLaughlin M (2008) Cost-effectiveness of nurse-led disease management for heart failure in an ethnically diverse urban community. Ann Intern Med 149:540-548

22. Maru S, Byrnes J, Carrington MJ, Chan YK, Thompson DR, Stewart S, Scuffham PA, WHICH? Trial Investigators (2015) Cost-effectiveness of home versus clinic-based management of chronic heart failure: extended follow-up of a pragmatic, multicentre randomized trial cohort - the WHICH? study (Which Heart Failure Intervention Is Most Cost-Effective \& Consumer Friendly in Reducing Hospital Care). Int J Cardiol 201:368-375. https://doi.org/10.1016/j.ijcard.2015.08.066

23. Mejia A, Richardson G, Pattenden J, Cockayne S, Lewin R (2014) Cost-effectiveness of a nurse facilitated, cognitive behavioural selfmanagement programme compared with usual care using a CBT manual alone for patients with heart failure: secondary analysis of data from the SEMAPHFOR trial. Int J Nurs Stud 51:1214-1220. https://doi.org/10.1016/j.ijnurstu.2014.01.009

24. Neumann A, Mostardt S, Biermann J, Gelbrich G, Goehler A, Geisler BP, Siebert U, Störk S, Ertl G, Angerrmann CE, Wasem J (2015) Cost-effectiveness and cost-utility of a structured collaborative disease management in the Interdisciplinary Network for Heart Failure (INH) study. Clin Res Cardiol 104:304-309. https://doi.org/ 10.1007/s00392-014-0781-4

25. Patel H, Shafazand $M$, Ekman I, Höjgård S, Swedberg K, Schaufelberger M (2008) Home care as an option in worsening chronic heart failure - a pilot study to evaluate feasibility, quality adjusted life years and cost-effectiveness. Eur J Heart Fail 10:675681. https://doi.org/10.1016/j.ejheart.2008.05.012

26. Reilly CM, Butler J, Culler SD, Gary RA, Higgins M, Schindler P, Butts B, Dunbar SB (2015) An economic evaluation of a self-care intervention in persons with heart failure and diabetes. J Card Fail 21:730-737. https://doi.org/10.1016/j.cardfail.2015.06.382

27. Cui Y, Doupe M, Katz A, Nyhof P, Forget EL (2013) Economic evaluation of Manitoba health lines in the management of congestive heart failure. Healthcare Policy 9(2):36-50

28. Zanaboni P, Landolina M, Marzegalli M, Lunati M, Perego G et al (2013) Cost-utility analysis of the EVOLVO study on remote monitoring for heart failure patients with implantable defibrillators: randomized controlled trial. J Med Internet Res 15(5):e106:1-11

29. Reed $\mathrm{S}$ et al (2010) Economic evaluation of the HF-ACTION (Heart Failure: A Controlled Trial Investigating Outcomes of Exercise Training) randomized controlled trial: an exercise training study of patients with chronic heart failure. Circ Cardiovasc Qual Outcomes 3:374-381
30. Sanders-van Wijk S, van Asselt A, Rickli H, Estlinbaum W, Erne P, Rickenbacher P, Vuillomenet A, Peter M, Pfisterer ME, Brunner-la Rocca HP, TIME-CHF Investigators (2013) Cost-effectiveness of $\mathrm{N}$-terminal pro-B-type natriuretic-guided therapy in elderly heart failure patients: results from TIME-CHF (Trial of Intensified versus Standard Medical Therapy in Elderly Patients with Congestive Heart Failure). JACC Heart Fail 1:64-71. https://doi.org/10.1016/ j.jchf.2012.08.002

31. Sánchez J et al (2010) Efficacy of peritoneal ultrafiltration in the treatment of refractory congestive heart failure. Nephrol Dial Transplant 25:605-610

32. Alehagen U, Rahmqvist M, Paulsson T, Levin LA (2008) Qualityadjusted life year weights among elderly patients with heart failure. Eur J Heart Fail 10:1033-1039. https://doi.org/10.1016/j.ejheart. 2008.07.015

33. Dolan P (1997) Modeling valuations for EuroQol health states. Med Care 35:1095-1108

34. Brazier J, Roberts J, Deverill M (2002) The estimation of a preference-based measure of health from the SF-36. J Health Econ 21:271-292

35. Franks P, Lubetkin E, Gold M, Tancredi D (2003) Mapping the SF12 to preference-based instruments: convergent validity in a lowincome, minority population. Med Care 41:1277-1283

36. Viney R, Norman R, King MT, Cronin P, Street DJ, Knox S, Ratcliffe J (2011) Time trade-off derived EQ-5D weights for Australia. Value Health 14:928-936. https://doi.org/10.1016/j.jval. 2011.04.009

37. Shaw JW, Johnson JA, Coons SJ (2005) US valuation of the EQ-5D health states: development and testing of the D1 valuation model. Med Care 43:203-220

38. Kind P (2003) Guidelines for value sets in economic non-economic studies using EQ-5D. In: Brooks R, Rabin R, de Charro F (eds) The measurement and valuation of health status using EQ-5D: a European perspective. Kluwer Academic Publishers, Dordrecht

39. Sandhu AT, Goldhaber-Fiebert JD, Owens DK, Turakhia MP, Kaiser DW, Heidenreich PA (2016) Cost-effectiveness of implantable pulmonary artery pressure monitoring in chronic heart failure. JACC Heart Fail 4:368-375. https://doi.org/10.1016/j.jchf.2015. 12.015

40. Scuffham PA, Whitty JA, Mitchell A, Viney R (2008) The use of QALY weights for QALY calculations: a review of industry submissions requesting listing on the Australian Pharmaceutical Benefits Scheme 2002-4. Pharmacoeconomics 26:297-310

41. Rowen D, Azzabi Zouraq I, Chevrou-Severac H, van Hout B (2017) International regulations and recommendations for utility data for health technology assessment. Pharmacoeconomics 35: 11-19. https://doi.org/10.1007/s40273-017-0544-y

42. Rector TS, Kubo S et al (1987) Patients' self-assessment of their congestive heart failure. Part 2: content, reliability and validity of a new measure, the Minnesota Living with Heart Failure Questionnaire. Heart Fail 3:198-209

43. Kularatna S, Byrnes J, Chan YK, Carrington MJ, Stewart S, Scuffham PA (2017) Comparison of contemporaneous responses for EQ-5D-3L and Minnesota Living with Heart Failure; a case for disease specific multiattribute utility instrument in cardiovascular conditions. Int J Cardiol 227:172-176. https://doi.org/10.1016/j. ijcard.2016.11.030

44. Mejhert M, Kahan T, Persson H, Edner M (2004) Limited long term effects of a management programme for heart failure. Heart. 90: 1010-1015

45. Dolan P, Sutton M (1997) Mapping visual analogue scale health state valuations onto standard gamble and time trade-off values. Soc Sci Med 44:1519-1530

46. Longworth L, Rowen D (2013) Mapping to obtain EQ-5D utility values for use in NICE health technology assessments. Value Health 16:202-210. https://doi.org/10.1016/j.jval.2012.10.010 
47. Feldman AM, de Lissovoy G, Bristow MR, Saxon LA, de Marco T, Kass DA, Boehmer J, Singh S, Whellan DJ, Carson P, Boscoe A, Baker TM, Gunderman MR (2005) Cost effectiveness of cardiac resynchronization therapy in the Comparison of Medical Therapy, Pacing, and Defibrillation in Heart Failure (COMPANION) trial. J Am Coll Cardiol 46:2311-2321. https://doi.org/10.1016/j.jacc. 2005.08.033

48. Moertl D, Steiner S, Coyle D, Berger R (2013) Cost-utility analysis of nt-probnp-guided multidisciplinary care in chronic heart failure. Int J Technol Assess Health Care 29:3-11

49. Brazier J, Yang Y, Tsuchiya A, Rowen DL (2010) A review of studies mapping (or cross walking) non-preference based measures of health to generic preference-based measures. Eur J Health Econ 11:215-225. https://doi.org/10.1007/s10198-009-0168-Z

50. Clarke A, Pulikottil-Jacob R, Connock M, Suri G, Kandala NB, Maheswaran H, Banner NR, Sutcliffe P (2014) Cost-effectiveness of left ventricular assist devices (LVADs) for patients with advanced heart failure: analysis of the British NHS bridge to transplant (BTT) program. Int J Cardiol 171:338-345. https://doi.org/10.1016/j. ijcard.2013.12.015

51. Yao G, Freemantle N, Calvert MJ, Bryan S, Daubert JC, Cleland JG (2007) The long-term cost-effectiveness of cardiac resynchronization therapy with or without an implantable cardioverter-defibrillator. Eur Heart J 28:42-51

52. Wailoo AJ, Hernandez-Alava M, Manca A, Mejia A, Ray J, Crawford B, Botteman M, Busschbach J (2017) Mapping to estimate health-state utility from non-preference-based outcome measures: an ISPOR good practices for outcomes research task force report. Value Health 20:18-27. https://doi.org/10.1016/j.jval.2016. 11.006
53. Petrou S, Rivero-Arias O, Dakin H, Longworth L, Oppe M, Froud R, Gray A (2015) Preferred reporting items for studies mapping onto preference-based outcome measures: the MAPS statement. J Med Econ 18:851-857. https://doi.org/10.3111/13696998.2015. 1070554

54. Ara R, Rowen D, Mukuria C (2017) The use of mapping to estimate health state utility values. Pharmacoeconomics 35:57-66. https:// doi.org/10.1007/s40273-017-0548-7

55. Sculpher M, Gafni A (2001) Recognizing diversity in public preferences: the use of preference sub-groups in cost-effectiveness analysis. Health Econ 10:317-324

56. Sculpher M, Gafni A (2002) Recognising diversity in public preferences: the use of preference sub-groups in cost-effectiveness analysis. Author's reply. Health Econ 11:653-654

57. Robinson A, Parkin D (2002) Recognising diversity in public preferences: the use of preference sub-groups in cost-effectiveness analysis. A response to Sculpher and Gafni. Health Econ 11:649-651

58. Cleland JG, Charlesworth A, Lubsen J, Swedberg K, Remme WJ, Erhardt L, di Lenarda A, Komajda M, Metra M, Torp-Pedersen C, Poole-Wilson PA, COMET Investigators (2006) A comparison of the effects of carvedilol and metoprolol on well-being, morbidity, and mortality (the "patient journey") in patients with heart failure: a report from the Carvedilol Or Metoprolol European Trial (COMET). J Am Coll Cardiol 47:1603-1611. https://doi.org/10. 1016/j.jacc.2005.11.069

Publisher's note Springer Nature remains neutral with regard to jurisdictional claims in published maps and institutional affiliations. 